\title{
Predictors of coronavirus disease 19 (COVID-19) pneumonitis outcome based on computed tomography (CT) imaging obtained prior to hospitalization: a retrospective study
}

\author{
Mohammad Mirza-Aghazadeh-Attari ${ }^{1,2,3} \cdot$ Armin Zarrintan $^{2,3,4} \cdot$ Nariman Nezami $^{3,5} \cdot$ Afshin Mohammadi $^{3,6}$. \\ Anita Zarrintan $^{3,7}$ - Iraj Mohebbi, ${ }^{3,8}$ Habibollah Pirnejad ${ }^{3,9}$ - Kamal Khademvatani ${ }^{3,10}$ - Zahra Ashkavand ${ }^{3,11}$. \\ Payman Forughi $^{3,7}$. Amin Arasteh ${ }^{1,2,3}$ • Javad Aghazadeh Attari ${ }^{3,7,11,12}$
}

Received: 15 June 2020 / Accepted: 29 July 2020 / Published online: 8 August 2020

(C) American Society of Emergency Radiology 2020

\begin{abstract}
Purpose Computed tomography (CT) has been utilized as a diagnostic modality in the coronavirus disease 19 (COVID-19), while some studies have also suggested a prognostic role for it. This study aimed to assess the diagnostic and prognostic value of computed tomography (CT) imaging in COVID-19 patients.

Methods This was a retrospective study of fifty patients with COVID-19 pneumonia. Twenty-seven patients survived, while 23 passed away. CT imaging was performed in all of the patients on the day of admission. Imaging findings were interpreted based on current guidelines by two expert radiologists. Imaging findings were compared between surviving and deceased patients. Lung scores were assigned to patients based on CT chest findings. Then, the receiver operating characteristic curve was used to determine cutoff values for lung scores.

Results The common radiologic findings were ground-glass opacities (82\%) and airspace consolidation (42\%), respectively. Air bronchogram was more commonly seen in deceased patients $(p=0.04)$. Bilateral and multilobar involvement was more frequently found in deceased patients ( $p=0.049$ and 0.014 , respectively). The mean number of involved lobes was $3.46 \pm 1.80$ lobes in surviving patients and $4.57 \pm 0.60$ lobes in the deceased patients $(p=0.009)$. The difference was statistically significant. The area under the curve for a lung score cutoff of 12 was 0.790 .

Conclusion Air bronchogram and bilateral and multilobar involvement were more frequently seen in deceased patients and may suggest a poor outcome for COVID-19 pneumonia.
\end{abstract}

Keywords COVID-19 · SARS-Cov-2 · Pneumonia $\cdot$ CT $\cdot$ Prognosis · Outcome

Mohammad Mirza-Aghazadeh-Attari, Armin Zarrintan and Javad Aghazadeh Attari contributed equally to this work.

Javad Aghazadeh Attari

m.aghazadeh96@gmail.com

1 Aging Research Institute, Tabriz University of Medical Sciences, Tabriz, Iran

2 Medical Radiation Research Center, Tabriz University of Medical Sciences, Tabriz, Iran

3 Department of Radiology, Neuro-Intervention and Neurologic Surgery, Urmia University of Medical Sciences, Urmia, Iran

4 Department of Radiology, Faculty of Medicine, Tabriz University of Medical Sciences, Tabriz, Iran

5 Division of Vascular and Interventional Radiology, Russell H. Morgan Department of Radiology and Radiological Science, The Johns Hopkins Medical Institutions, Baltimore, MD, USA
6 Department of Radiology, Urmia University of Medical Sciences, Urmia, Iran

7 Department of Internal Medicine, Tabriz University of Medical Sciences, Tabriz, Iran

8 Social Determinants of Health Research Center, Urmia University of Medical Sciences, Urmia, Iran

9 Health Information Technology Department, Urmia University of Medical Sciences, Urmia, Iran

10 Department of Cardiology, Faculty of Medicine, Urmia University of Medical Sciences, Urmia, Iran

11 Department of Regenerative and Cancer Cell Biology, Albany Medical College, Albany, NY, USA

12 Faculty of Medicine, UMSU Campus, Daneshgah Avenue, Urmia, Iran 


\section{Introduction}

The coronavirus disease 19 (COVID-19) emerged in late 2019 in the Chinese city of Wuhan. This virus was the third coronavirus resulting in an epidemic, while the previous ones being the severe acute respiratory syndrome virus and the Middle Eastern respiratory syndrome virus [1-3]. The condition is characterized by lower respiratory involvement, causing viral pneumonia, causing clinical signs and symptoms such as fever, coughs, and systemic symptoms of inflammation [4]. The disease has caused a significant number of deaths around the world. Most of these patients die from severe acute respiratory syndrome, but new evidence suggests that cardiovascular involvement and systemic inflammatory responses causing sepsis may also be involved [5]. Epidemiologic data suggest that most patients dying are those with old age or pre-existing medical conditions and those with pre-existing needs for medical attention [6]. To better allocate resources and to avoid overtreatment or undertreatment, some scholars have focused on determining prognostic factors of the disease, such as laboratory tests, the existence of pre-existing conditions, and, most recently, imaging findings [7]. First reports have shown that computed tomography (CT) has a high sensitivity in diagnosing the disease and can even show abnormalities before molecular assay shows positive results. More so, the extent of involvement may help in clinical decision-making in asymptomatic patients or those with mild to moderate symptoms $[8,9]$. It is worthy to mention that any criteria or factors introduced have to be simple to interpret and should be applied in the over-crowded wards or emergency departments [10].

Currently, little evidence exists regarding the prognostic features of CT in COVID-19 patients [11], and limited clinical experience is conveyed by available scientific literature [12]. Therefore, this study aimed to assess imaging findings in COVID-19 patients and compare CT chest findings in patients who were recovering and who passed away from COVID-19 pneumonia.

\section{Materials and methods}

\section{Study design}

This was a retrospective study conducted in tertiary medicaleducational centers of a consolidated health system, providing service to approximately 4 million people. All patients involved in the study presented to these centers between February 25 and April 15, 2020.

The study was approved by the local ethics board of the institution in which it was performed (IR.UMSU. REC.1399.016). All patients being included in the study had signed informed written consent. The study complied with the latest Helsinki declaration.

\section{Study population}

All patients enrolled in this study had presented between February 25 and April 15 with symptoms suggestive of COVID-19. All patients had a positive polymerase chain reaction (PCR) test. Past medical records of all patients were reviewed before enrolling in the study. Patients were included in this study and were then followed up until discharge or death. Inclusion criteria consisted of patients with a positive molecular assay for COVID-19 who were hospitalized, who either passed away from the disease or who recovered, and who were discharged from the hospitals. Exclusion criteria consisted of those patients with terminal medical illnesses, such as stage 4 cancers, heart failure, and acute kidney failure; trauma patients; and patients undergoing surgery 1 month before being considered for inclusion. The discharge processes were planned based on the criteria suggested by the Centers for Disease Control (CDC) of the USA and China [13].

Patients underwent an imaging protocol based on the World Health Organization (WHO) guidelines and institutional guidelines [14]. Imaging was done after consultation with internists. CT examinations of all patients had been performed on the initial day of admission.

Patients were classified into two groups: the surviving group who were cured after COVID-19 and the deceased group who passed away from COVID-19 pneumonia.

\section{COVID-19 test}

All patients had a molecular assay done based on the latest guidelines proposed by the World Health Organization [15]. All of the patients included in the study had a positive PCR test, which was done on a sample of nasopharynx and oropharynx. The kits used for the diagnosis were approved by the Center for Disease Control of China and the World Health Organization. The specificity of the test was assumed to be $100 \%$, and previous studies had shown that the sensitivity of the test was $60 \%$. [16].

\section{Imaging protocol}

CT examinations of all patients had been performed prior to admission in the emergency department, using Siemens SOMATOM (Hannover, Germany) scanner based on the following protocol: low-dose mode, automatic tube current modulation with a voltage of $115-120 \mathrm{kV}$, mAs 250 , matrix size of $512 \times 512$ increments, and thickness of $1.5-2 \mathrm{~mm}$. CT images were reconstructed in both transverse and sagittal planes. No contrast media was injected. 


\section{Image analysis}

$\mathrm{CT}$ chest images were reviewed on an online picture archive and communication system (PACS). Review and analysis of the $\mathrm{CT}$ images were done by two independent radiologists with at least 10 years of experience in thoracic imaging. Images were assessed based on a checklist adapted from the recommendations of the Radiological Society of North America (RSNA) and by Kanne et al. [17, 18]. Bilateral involvement was defined as both of the lungs showing abnormal radiologic findings, and multifocal involvement was defined as having multiple lesions in at least two lobes of either of the lungs. Different radiologic signs and patterns of involvement were recorded for all patients. Lung score was determined based on the following [19]: 0: no involvement, 1: less than $5 \%$ of involvement, $2: 6-25 \%$ involvement, $3: 26-50 \%$ involvement, 4: 51-75\% involvement, and 5: involvement more than $75 \%$. In cases of disagreement, a third radiologist reviewed the imaging, and then, a consensus decision was made. The radiologists were blinded to the PCR results and clinical outcomes.

\section{Statistical analysis}

The statistical analysis was performed with the help of the SSPS statistical software version 23 (New York, USA). Continuous variables were tested for normal distribution with the Kolmogorov-Smirnov and Shapiro-Wilk tests. The parametric data are presented as median or mean \pm standard deviation. The non-parametric data are presented in number and percentage (\%). Descriptive statistics were used for descriptive analysis and independent sample $t$ test, Pearson's chisquare test, and Fisher's exact test were utilized to analyze the variables and their relations. The receiver operating characteristics (ROC) curve was utilized to calculate the area under the curve and determine a cutoff point for lung scores. An area under the curve between 1 and 0.9 was considered excellent, 0.9 to 0.8 was regarded to be good, 0.8 to 0.7 was fair, and 0.7 to 0.6 was a poor predictor of mortality [20]. Relative risk (RR) was also calculated regarding the number of lobes involved and clinical outcome (survival or death). The power of the study was calculated by using a repeated measure general linear model and by calculating Pillai's trace, which was calculated to be $80 \%$, and a $p$ value of less than 0.05 was considered statistically significant.

\section{Results}

A total of 50 patients were included in this study, of which 27 survived and 23 passed away. Of all the patients, 23 were female and 27 were male. In the first group, 15 were female and 12 were male, while in the second group, eight were female and 15 were male. The difference between the two groups was not statistically significant $(p=0.142$ ). The mean age of all of the patients being included in the study was 65.4 \pm 16.77 years old. The mean age of patients surviving the disease was $60.93 \pm 15.94$ years, and in the patients dying was $70.65 \pm 16.51$ years old, with the difference being significant $(p=0.04)$. Pre-existing conditions for each group of patients and clinical signs are represented in Tables 1 and 2.

The imaging findings of patients being included in the study are presented in Table 3 . The mean number of segments involved in the surviving patients was $3.46 \pm 1.8$ lobes, while it was $4.57 \pm 0.6$ in those dying of the disease, with the difference being significant $(p=0.009)$. Specific CT chest imaging findings of patients with COVID-19 pneumonia are demonstrated in Figs. 1 and 2; Fig. 1 shows septal thickening and crazy-paving pattern and demonstrates bilateral air bronchogram and reticulonodular lesions, and Fig. 2 shows atelectasis and peribronchovascular involvement, and typical bilateral ground-glass opacities and airspace consolidation.

A comparison of imaging findings between the two groups is shown in Table 2. The frequency of bilateral lung involvement was significantly higher in the deceased group between the two groups $(p=0.04)$. Air bronchogram was significantly more common in the deceased group $(p=0.03)$. Multifocal involvement was also significantly more in this group ( $p=$ $0.014)$. The number of involved lobes was significantly higher in the deceased group $(p=0.001)$. The mean lung score was
Table 1 Clinical signs and symptoms and pre-existing medical conditions in patients

\begin{tabular}{llcc}
\hline Clinical signs and symptoms & Surviving patients & Deceased patients & Total \\
\hline Fever & $8(29.6 \%)$ & $9(39.1 \%)$ & $17(34 \%)$ \\
Cough & $11(40.7 \%)$ & $11(47.8 \%)$ & $22(44 \%)$ \\
Dyspnea & $11(40.7 \%)$ & $14(60.8 \%)$ & $25(50 \%)$ \\
Chest pain & $2(7.1 \%)$ & $3(13.0 \%)$ & $5(10 \%)$ \\
Nausea & $2(7.1 \%)$ & $1(4.3 \%)$ & $3(6 \%)$ \\
Pharyngitis & $0(0.0 \%)$ & $4(17.3 \%)$ & $4(8 \%)$ \\
Myalgia & $0(0.0 \%)$ & $2(8.6 \%)$ & $2(4 \%)$ \\
\hline
\end{tabular}


Table 2 Pre-existing conditions of patients being included in the study

\begin{tabular}{lllc}
\hline Pre-existing conditions & Surviving patients & Deceased patients & Total \\
\hline Acute coronary syndrome & $4(14.8 \%)$ & $8(34.7 \%)$ & $12(24 \%)$ \\
Hypertension & $4(14.8 \%)$ & $6(26.0 \%)$ & $10(20 \%)$ \\
Pre-existing respiratory condition & $1(3.7 \%)$ & $3(11.1 \%)$ & $4(8 \%)$ \\
Diabetes & $4(14.8 \%)$ & $5(21.7 \%)$ & $9(18 \%)$ \\
Chronic kidney disease & $1(3.7 \%)$ & $2(8.6 \%)$ & $3(6 \%)$ \\
\hline
\end{tabular}

statistically higher in the deceased group compared with that in the surviving group $(15.80 \pm 4.52$ vs $9.00 \pm 6.14$, respectively; $p=0.0001)$. The ROC curve for a lung score cutoff of 12 is shown in Fig. 3. The area under the curve was 0.790 , which is considered to be a fair predictor of mortality in patients with a lung score higher than 12 . The RR of surviving from COVID19 pneumonia was 2.150 (95\% CI: 1.560-2.962) times higher for COVID-19 patients with two or less lobe involved compared with patients with three or more lobes involved. The RR of surviving in patients with lung score of less than 12 was 2.414 (95\% CI: 1.14-5.091) times higher than patients with a lung score equal to 12 or higher.

Of the 23 patients dying from the disease, 11 were hospitalized in an intensive care unit (ICU) and nine were intubated. Of the 27 patients surviving, nine had to be hospitalized in an ICU, with 3 of them being intubated. A retrospective study of all of the medical records revealed that none of the patients in the surviving group had any diagnostic workup done for other pulmonary manifestations of COVID-19, such as alveolar hemorrhage or pulmonary thromboembolism (PTE). In the other group, two patients underwent CT angiography to rule out PTE, as both had deteriorated unexpectedly. PTE was ruled out for both patients. One patient in the dying group developed alveolar hemorrhage during hospitalization and was treated by interventional radiologists.

Our retrospective investigation also revealed that two patients from each group developed healthcare-associated pneumonia (HCAP). All four patients had methicillin-resistant Staphylococcus aureus (MRSA) isolated from their respiratory tracts.

None of the patients in the surviving group developed acute kidney injury, while six patients from the 23 dying developed
Table 3 Radiologic signs and patterns of involvement in two groups of patients (significant variables are italicized)

\begin{tabular}{lccc}
\hline & Surviving patients & Deceased patients & $p$ value \\
\hline Radiologic signs & & & \\
Ground-glass opacities & $22(81.4 \%)$ & $19(82.6 \%)$ & 0.71 \\
Crazy-paving & $5(18.51 \%)$ & $6(26.0 \%)$ & 0.5 \\
Airspace consolidation & $9(33.3 \%)$ & $12(52.1 \%)$ & 0.1 \\
Septal thickening & $13(48.1 \%)$ & $7(30.4 \%)$ & 0.38 \\
Reticulonodular lesions & $4(14.8 \%)$ & $2(8.6 \%)$ & 0.68 \\
Tree-in-bud & $0(0.0 \%)$ & $0(0.0 \%)$ & $\mathrm{n} / \mathrm{a}$ \\
Peribronchovascular involvement & $4(14.8 \%)$ & $4(17.3 \%)$ & 0.71 \\
Bronchiectasis & $1(3.7 \%)$ & $2(8.6 \%)$ & 0.56 \\
Cavitation & $0(0.0 \%)$ & $0(0.0 \%)$ & $\mathrm{n} / \mathrm{a}$ \\
Air bronchogram & $8(29.6 \%)$ & $13(56.5 \%)$ & 0.04 \\
Pattern of involvement & & & \\
Bilateral & $23(85.1 \%)$ & $23(100 \%)$ & 0.049 \\
Unilateral & $1(3.7 \%)$ & $0(0.0 \%)$ & 0.371 \\
Unifocal & $3(11.1 \%)$ & $0(0.0 \%)$ & 0.24 \\
Multifocal & $21(77.7 \%)$ & $23(100 \%)$ & 0.014 \\
Peripheral & $19(70.3 \%)$ & $15(65 \%)$ & 0.9 \\
Central & $11(40.7 \%)$ & $14(60.8 \%)$ & 0.154 \\
Anterior & $9(33.3 \%)$ & $9(39.1 \%)$ & 0.52 \\
Posterior & $21(77.7 \%)$ & $20(86.9 \%)$ & 0.26 \\
Mediastinal lymphadenopathy & $3(11.1 \%)$ & $4(17.3 \%)$ & 0.68 \\
Pleural effusion. & $1(3.7 \%)$ & $5(21.7 \%)$ & 0.075 \\
\hline
\end{tabular}




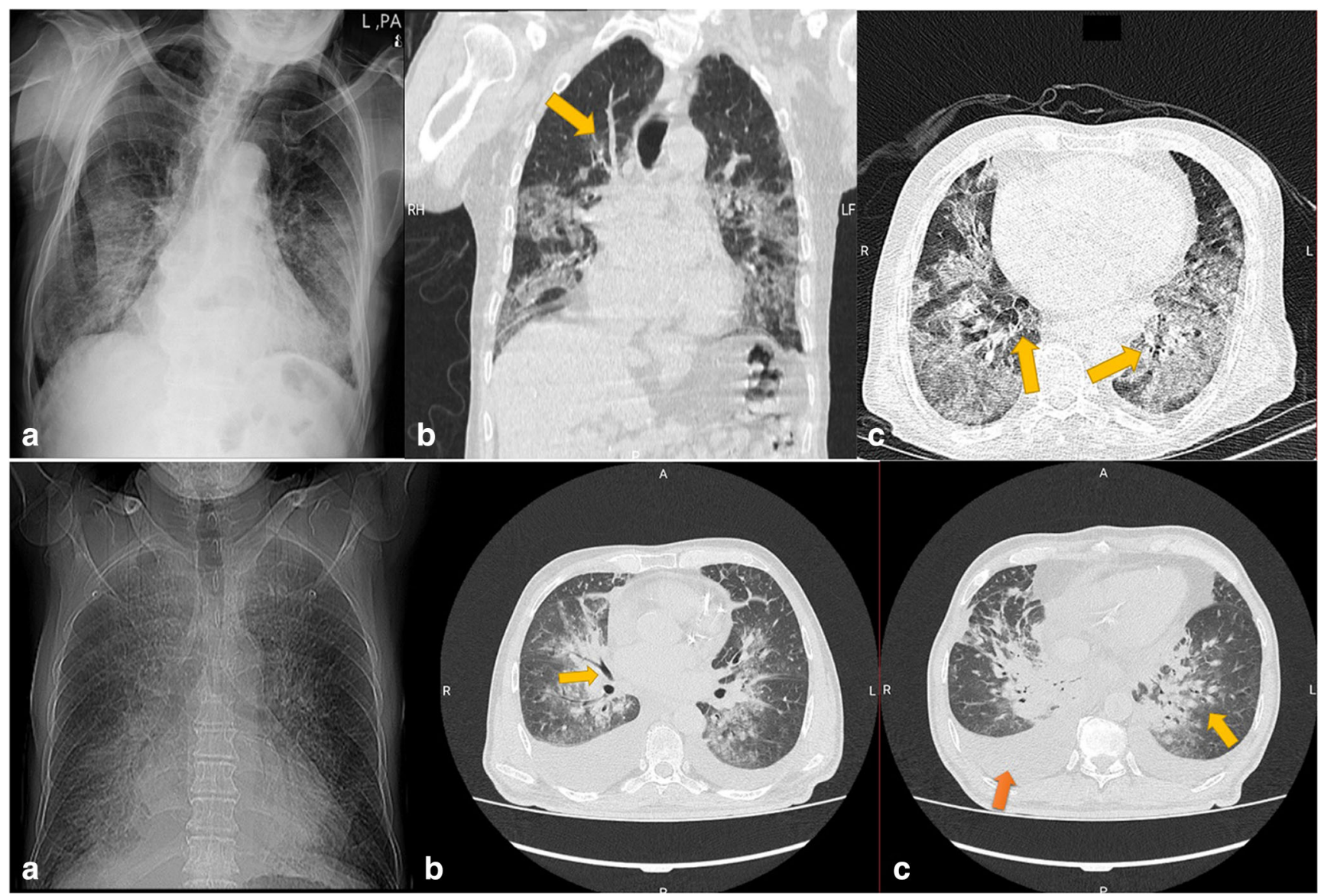

Fig. 1 Upper row. Right a A 114-year-old male passed away from COVID-19 pneumonia. a Posterior anterior chest x-ray shows opacities in the lower lobes of both lungs. Center b Coronal CT image of the same patient, showing multilobar ground-glass opacities and septal thickening (yellow arrow). Left c Axial cut from the same patient shows multifocal ground-glass opacities and crazy-paving, characterized by a superimposed interstitial thickening on ground-glass opacities (yellow arrow). Down row. Right a A 60-year-old man passing away from COVID-19. a PA chest X-ray on admission, showing haziness in central regions. Center $\mathbf{b}$ An axial cut of the same patient. The yellow arrow marks an air bronchogram. Left c Axial cut showing reticulonodular opacities in both lungs, with the predominance of the left one (yellow arrow). This patient had a severe case of pleural effusion, most prominent on the right side (red arrow)
AKI during hospitalization or on presentation. Liver enzymes were elevated in 5 patients in the surviving group, while nine patients had elevated liver enzymes in the other group.

Of the patients surviving, 21 received hydroxychloroquine, two received oseltamivir, and 2 received lopinavir and ritonavir. From those dying, ten received hydroxychloroquine, seven received oseltamivir, two received lopinavir-ritonavir, and one patient received ritonavir. None of the patients being included in the study received immunosuppressant agents of any kind.

\section{Discussion}

Our findings showed that bilateral and multifocal involvement was significantly more common in deceased patients. Furthermore, the number of involved lobes and lung score in deceased patients were significantly higher, and air bronchogram was more commonly observed among deceased patients. Lung score of more than 12 was a good predictor of mortality in patients with COVID-19 pneumonia.

Since the beginning of the COVID-19 pandemic, a timely diagnosis has been an important concern. Systemic reviews of the evidence available suggest that CT imaging may have a higher sensitivity compared with molecular assays in countries with a high prevalence of the disease [21]. A retrospective study performed on 11 patients with COVID-19 pneumonia showed that peripheral ground-glass opacities were seen in all of the patients. Furthermore, compared with non-COVID19 pneumonia, some radiologic signs, including air bronchogram and reticular patterns, were seen significantly more common in COVID-19 pneumonia [22]. Other findings such as centrilobular nodules, tree-in-bud appearance, and subpleural linear opacities were more common in nonCOVID-19 pneumonia. Interestingly, involvement was more severe in the COVID-19 group, with the mean number of involved lobes and segments being significantly higher ( $p=0.000$ and $p=0.000$ respectively). 


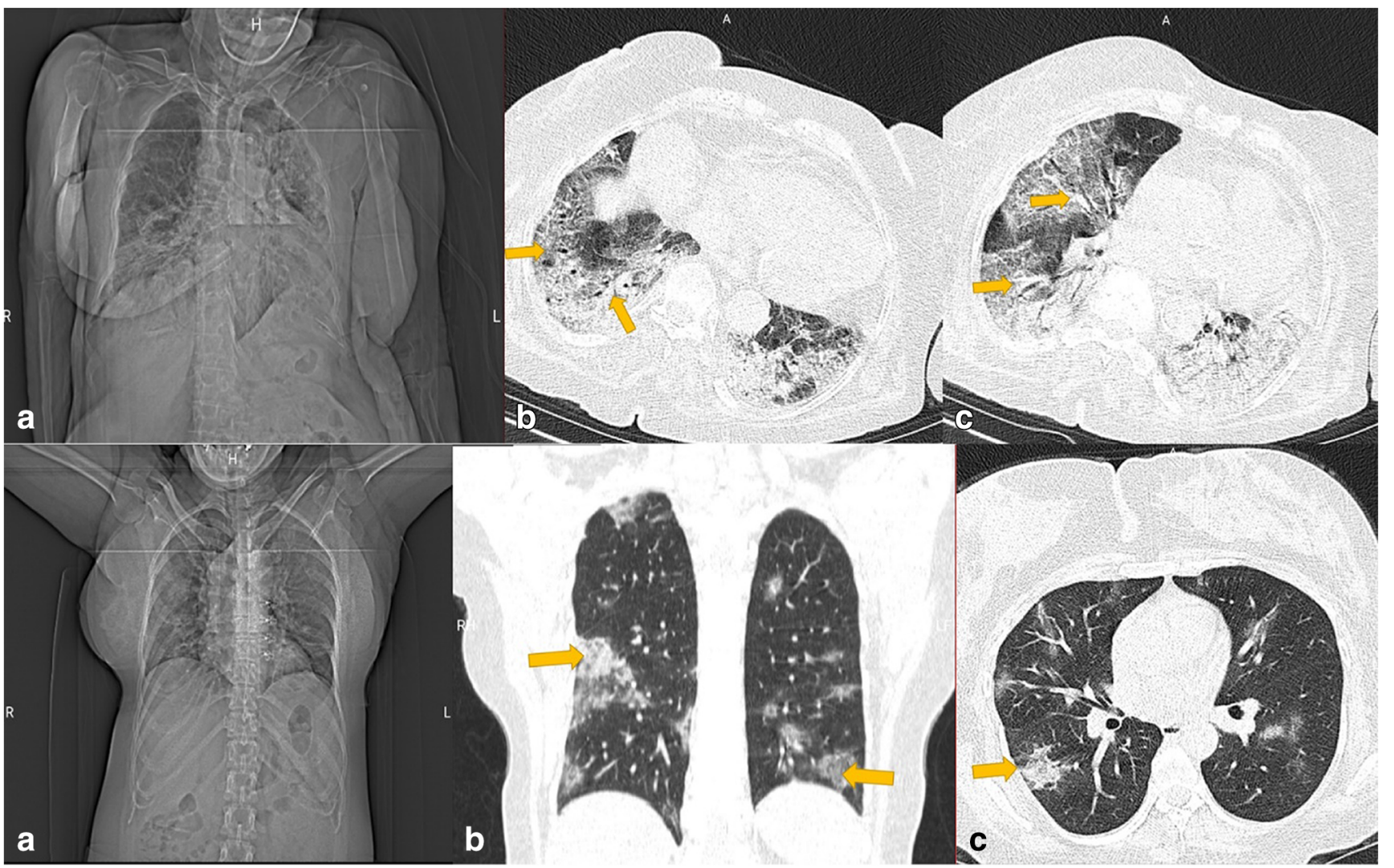

Fig. 2 Upper row. An 83-year-old woman who expired from COVID-19. Right a PA chest x-ray of the patient, showing haziness and opacities in both lungs, predominantly the basal section of each lung. Center b Axial $\mathrm{CT}$ imaging showing atelectasis (yellow arrow) and diffuse involvement of both lungs. Left $\mathbf{c}$ Peribronchovascular involvement in the same patient. Down row. A 36-year-old female patient who survived COVID-19

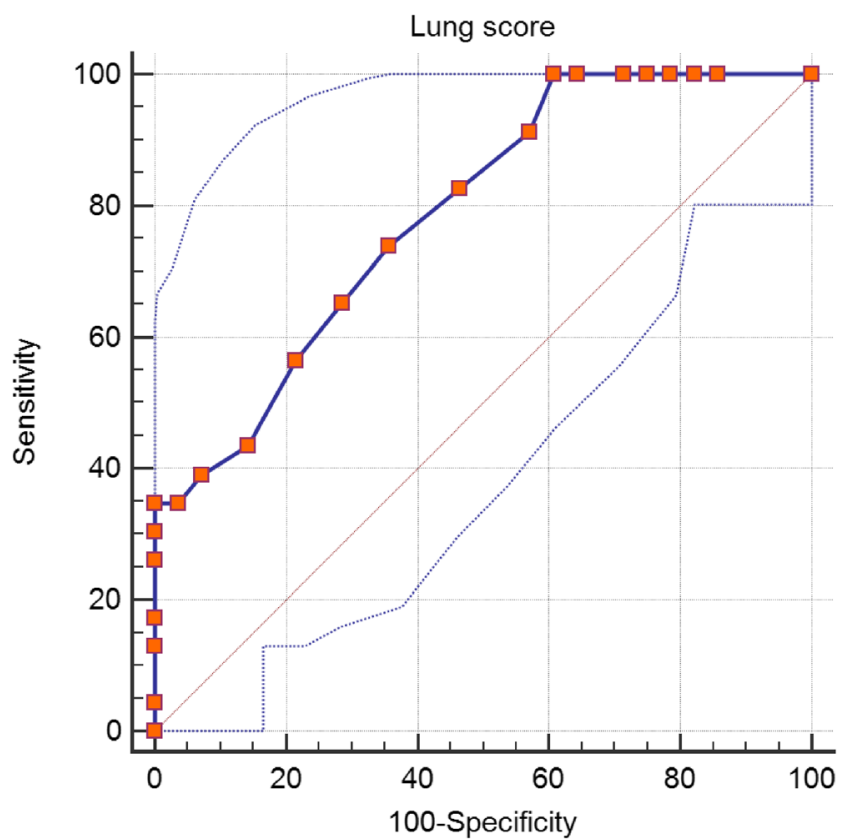

Fig. 3 ROC curve for a lung score of 12 . The area under the curve was 0.790 (95\% CI: $0.669-0.911$ ) and was hospitalized for 5 days. Right a PA chest x-ray of the patient. Center b Typical presentation of COVID-19, peripheral, multifocal ground-glass opacities (examples are shown by the yellow arrow). Left c Airspace consolidation is shown in the posterior segment of the right lung (yellow arrow)

Another clinical concern is screening for COVID-19 patients who are more likely to require intensive medical attention, as the large number of patients presenting to medical centers has overwhelmed the available human resources and existing medical infrastructure [23]. Based on currently available data, a wide range of clinical data, lab test results, and imaging findings are being considered for prognostic markers of the disease. The neutrophil-to-lymphocyte ratio (NLR), lymphocyte-to-monocyte (MON) ratio, platelet-tolymphocyte ratio (PLR), and C-reactive protein have been proposed as prognostic factors in COVID-19 patients. The HR for NLR of 3.3 in predicting severe COVID-19 pneumonia was 2.46 (95\% CI: 1.98-4.57) [24].

Early evidence from the application of imaging as a predictor of disease severity was introduced by Colombi et al. [25]. In their study, a total of 236 patients were included. One hundred and eight patients were in the ICU/death group, and the rest were patients who were hospitalized in non-intensive wards of the hospital. They found that incorporating clinical data and radiologic factors such as well-aerated lung parenchyma (assessed both visually and software) had a sensitivity of $75 \%$ (95\% CI: $66-83 \%$ ), a specificity of $81 \%$ (95\% CI: $73-$ 
$88 \%$ ), a positive predictive value of $77 \%$ (95\% CI: 69-83\%), and negative predictive value of $79 \%$ (95\% CI: 74-84\%), with an area under the curve of 0.86 (95\% CI: 0.81-0.90) ( $p$ val$\mathrm{ue}=0.04)$. More so, patients with grave clinical outcomes had a higher number of mean lobes involved.

Another cross-sectional study on 120 patients compared imaging findings of those hospitalized in routine wards and intensive care unit (ICU) who survived and those who died following hospitalization. The percentage of total lung involvement calculated by visual assessment by the radiologist for patients expiring or being hospitalized in the intensive care unit (ICU) exceeded that of patients hospitalized in the nonICU floor. The frequency of pleural effusions, crazy-paving, and air bronchograms was significantly higher in the patients who expired from COVID-19 pneumonia and hospitalized in the ICU, compared with that in the other patients. This study's findings were similar to our findings, although we included more patients dying from COVID-19, which could justify more severe radiologic findings witnessed in our study. Additionally, bilateral lung involvement with multifocal lesions was more common in deceased patients [26].

Another characteristic of CT imaging is the fact that studies have shown that it can differentiate between various stages of the disease [19]. In a retrospective study on 21 patients with COVID-19, four distinct stages were defined for disease progression. The first stage was characterized by abundant groundglass opacities, and the frequency of this finding decreased in higher stages. In stages four and three, the most common finding was consolidation, and stage two was characterized by a transition from ground-glass opacities to consolidations. The most number of involved lobes was seen in stage three of COVID-19 [27]. Studies suggest that abnormal patterns of involvement, such as co-appearance of crazy-paving, linear opacities, and consolidations in early stages of the disease, may be associated with either rapid progression and poor prognosis or shorter disease course with good prognosis [28].

Serial CT imaging may prove beneficial in monitoring disease progression, especially in cases with rapid clinical deterioration. Furthermore, serial imaging can determine the extent of damage to the lung tissue after the resolution of clinical signs and symptoms [29].

In our study, survivors had a significantly lower age than non-survivors, and fewer of them had pre-existing conditions. Older age is associated with irreversible damage to the cellular homeostasis and reduced functionality of body organs, which reduces an organism's capability to efficiently counter-react to external stimuli [30]. The same can be said for those with irreversible loss of function in critical organs such as the kidneys, liver, and heart. COVID-19 burdens the body with reduced oxygenation, which contributes to the dysfunction of already compromised organs. Exaggerated immune responses to the virus and initiation of a multisystem inflammatory syndrome further reduce the capability of those organs to maintain functionality [31]. Large-scale clinical studies suggest that old age and specific pre-existing conditions are the most important risk factors for mortality due to COVID-19. Thus, the elderly, who are usually affected by multiple underlying conditions, should be considered high-risk patients and be treated immediately [32]. In our study, most of the patients received hydroxychloroquine, an anti-malarial agent, or antivirals such as lopinavir/ritonavir and oseltamivir. Based on the already existing evidence, none of these agents has shown beneficence in large-scale studies, and the results of the few studies which have shown efficacy for any of the above agents have not been reproduced in larger scale studies [33]. Immunosuppressants are agents that have shown promise in the treatment of COVID-19, especially in the gravely ill [34]. Our subjects did not receive immune suppressants as they were infected by the virus in the early stages of the epidemic when evidence was not abundant. Currently, a wide range of immunosuppressants and novel antiviral agents such as remdesivir and favipiravir are being considered for treatment [35].

This study had several limitations. Only a group of 50 COVID-19 patients were evaluated in this study, which could limit the generalizability of our findings. This is the common challenge with the majority of studies conducted on COVID-19 pneumonia today. A large-scale multicenter study could confirm these findings. Furthermore, our study was more focused on specific radiologic signs, the number of lobes involved, and the pattern of involvement. This imaging approach and definition is easily perceptible by clinicians in daily clinical practice, while more sophisticated approaches involving lung severity score calculation or application of artificial intelligence algorithms could result in more accurate results.

In conclusion, bilateral lung involvement, the involvement of 3 or more lobes, lung score of more than 12, and air bronchogram on CT chest are associated with a higher likelihood of mortality. These imaging findings should prompt either close monitoring or listing the patient with urgent medical interventions.

\section{Main points}

1 Air bronchogram and bilateral and multifocal involvement may be a predictor of a worse clinical outcome in COVID19.

2 Lung score was significantly higher in patients dying from COVID-19 compared with that in those surviving.

3 The area under the curve was 0.790 (95\% CI: 0.669 $0.911)$, suggesting a fair predictive value for the curve.

Authors' contributions MMAA: Drafting of manuscript, original idea, statistical analysis.

ArZ: Interpretation of imaging findings, original idea, drafting of the manuscript. 
NN: Final edit, drafting of the manuscript, literature review, interpretation of imaging findings.

AM: Final edit, drafting of the manuscript, literature review.

AnZ: Data acquisition, drafting of the manuscript, final edit.

IM: Data acquisition, literature review, final edit.

HP: Data acquisition, literature review, final edit.

KK: Data acquisition, literature review, final edit.

ZA: Literature review, final edit, drafting of manuscript.

PF: Data acquisition, drafting of the manuscript, interpretation of laboratory data.

AA: Data acquisition, drafting of the manuscript, interpretation of laboratory data.

JAA: Drafting of manuscript, original idea, statistical analysis, interpretation of imaging findings.

Funding information This study was funded by Urmia University of Medical Sciences and Tabriz University of Medical Sciences.

Data availability All data will be made available based on reasonable request.

\section{Compliance with ethical standards}

Conflict of interest The authors declare that they have no conflict of interest.

Ethical approval The study was approved by the local ethics committee at Urmia University of Medical Sciences ((IR.UMSU.REC.1399.016).

Consent to participate All included patients signed written informed consent notes.

Consent for publication Consent was obtained from all patients.

\section{References}

1. Aghazadeh-Attari J, Mohebbi I, Mansorian B, Ahmadzadeh J, Mirza-Aghazadeh-Attari M, Mobaraki K, Oshnouei S (2018) Epidemiological factors and worldwide pattern of Middle East respiratory syndrome coronavirus from 2013 to 2016. Int J Gen Med 11:121-125

2. Xu Z, Shi L, Wang Y, Zhang J, Huang L, Zhang C, Liu S, Zhao P, Liu H, Zhu L, Tai Y, Bai C, Gao T, Song J, Xia P, Dong J, Zhao J, Wang FS (2020) Pathological findings of COVID-19 associated with acute respiratory distress syndrome. Lancet Respir Med 8(4): $420-422$

3. Ahmadzadeh J, Mobaraki K, Mousavi SJ, Aghazadeh-Attari J, Mirza-Aghazadeh-Attari M, Mohebbi I (2020) The risk factors associated with MERS-CoV patient fatality: a global survey. Diagn Microbiol Infect Dis 96(3):114876

4. Shi Y, Wang Y, Shao C, Huang J, Gan J, Huang X et al (2020) COVID-19 infection: the perspectives on immune responses. Nature Publishing Group, Berlin

5. Tufan A, Güler AA, Matucci-Cerinic M (2020) COVID-19, immune system response, hyperinflammation and repurposing antirheumatic drugs. Turk J Med Sci 50(SI-1):620-632

6. Surveillances V (2020) The epidemiological characteristics of an outbreak of 2019 novel coronavirus diseases (COVID-19) - China, 2020. China CDC Wkly 2(8):113-122

7. Zhao X, Zhang B, Li P, Ma C, Gu J, Hou P, et al (2020) Incidence, clinical characteristics and prognostic factor of patients with COVID-19: a systematic review and meta-analysis. medRxiv
8. Ayebare RR, Flick R, Okware S, Bodo B, Lamorde M (2020) Adoption of COVID-19 triage strategies for low-income settings. Lancet Respir Med 8(4):e22

9. Mohammadi A, Mohebbi I, khademvatani K et al (2020) Clinical and radiological characteristics of pediatric patients with COVID19: focus on imaging findings. Jpn J Radiol. https://doi.org/10. 1007/s11604-020-01003-6

10. Ala A, Shams Vahdati S, Ghaffarzad A, Mousavi H, MirzaAghazadeh-Attari M (2018) National emergency X-radiography utilization study guidelines versus Canadian C-Spine guidelines on trauma patients, a prospective analytical study. PLoS ONE 13(11): e0206283. https://doi.org/10.1371/journal.pone.0206283

11. Chen X, Liu S, Zhang C, Pu G, Sun J, Shen J, Chen Y (2020) Dynamic chest CT evaluation in three cases of 2019 novel coronavirus pneumonia. Arch Iran Med 23(4):277-280

12. Wei J, Xu H, Xiong J, Shen Q, Fan B, Ye C, Dong W, Hu F (2020) 2019 novel coronavirus (COVID-19) pneumonia: serial computed tomography findings. Korean J Radiol 21(4):501-504

13. Control CfD, Prevention (2020) Discontinuation of transmissionbased precautions and disposition of patients with COVID-19 in healthcare settings (Interim Guidance)

14. Organization WH (2020) Clinical management of severe acute respiratory infection when novel coronavirus $(\mathrm{nCoV})$ infection is suspected: interim guidance, January 25 2020. World Health Organization;

15. Organization WH (2020) Coronavirus disease 2019 (COVID-19): situation report, 72

16. Organization $\mathrm{WH}$ (2020) Laboratory testing for coronavirus disease 2019 (COVID-19) in suspected human cases: interim guidance, March 2 2020. World Health Organization;

17. Kanne JP (2020) Chest CT Findings in 2019 Novel Coronavirus (2019-nCoV) Infections from Wuhan, China: Key Points for the Radiologist. Radiology 295(1):16-17. https://doi.org/10.1148/ radiol.2020200241

18. Kanne JP, Little BP, Chung JH, Elicker BM, Ketai LH (2020) Essentials for Radiologists on COVID-19: An Update-Radiology Scientific Expert Panel. Radiology 296(2):E113-E114. https://doi. org/10.1148/radiol.2020200527

19. Bernheim A, Mei X, Huang M et al (2020) Chest CT Findings in Coronavirus Disease-19 (COVID-19): Relationship to Duration of Infection. Radiology 295(3):200463. https://doi.org/10.1148/ radiol.2020200463

20. Obuchowski NA, Bullen JA (2018) Receiver operating characteristic (ROC) curves: review of methods with applications in diagnostic medicine. Phys Med Biol 63(7):07TR1

21. Kim H, Hong H, Yoon SH (2020) Diagnostic performance of CT and reverse transcriptase-polymerase chain reaction for coronavirus disease 2019: A meta-analysis [published online ahead of print, 2020 Apr 17]. Radiology 201343. https://doi.org/10.1148/radiol. 2020201343

22. Cheng Z, Lu Y, Cao Q et al (2020) Clinical features and chest CT manifestations of coronavirus disease 2019 (COVID-19) in a single-center study in Shanghai, China. AJR Am J Roentgenol 215(1):121-126. https://doi.org/10.2214/AJR.20.22959

23. Poole DN, Escudero DJ, Gostin LO, Leblang D, Talbot EA (2020) Responding to the COVID-19 pandemic in complex humanitarian crises. Int J Equity Health 19(1):1-2

24. Yang AP, Liu JP, Tao WQ, Li HM (2020) The diagnostic and predictive role of NLR, d-NLR and PLR in COVID-19 patients. Int Immunopharmacol 84:106504. https://doi.org/10.1016/j.intimp. 2020.106504

25. Colombi D, Bodini FC, Petrini M et al (2020) Well-aerated lung on admitting chest CT to predict adverse outcome in COVID-19 pneumonia. Radiology 296(2):E86-E96. https://doi.org/10.1148/radiol. 2020201433 
26. Tabatabaei SMH, Talari H, Moghaddas F, Rajebi H (2020) Computed tomographic features and short-term prognosis of coronavirus disease 2019 (COVID-19) pneumonia: a single-center study from Kashan, Iran. Radiol Cardiothorac Imaging 2(2): e200130

27. Pan F, Ye T, Sun P et al (2020) Time course of lung changes at chest CT during recovery from coronavirus disease 2019 (COVID19). Radiology 295(3):715-721. https://doi.org/10.1148/radiol. 2020200370

28. Ding X, Xu J, Zhou J, Long Q (2020) Chest CT findings of COVID-19 pneumonia by duration of symptoms. Eur J Radiol 127:109009. https://doi.org/10.1016/j.ejrad.2020.109009

29. Tianhe Ye, Yanqing Fan, Jiacheng Liu et al. (2020) Follow-up Chest CT findings from discharged patients with severe COVID19: an 83-day observational study, 12 May 2020, PREPRINT (Version 1) available at Research Square. https://doi.org/10. 21203/rs.3.rs-27359/v1

30. Mohammadzadeh A, Mirza-Aghazadeh-Attari M, Hallaj S, Saei AA, Alivand MR, Valizadeh A, Yousefi B, Majidinia M (2019) Crosstalk between P53 and DNA damage response in ageing. DNA Repair 80:8-15
31. Mason RJ (2020) Pathogenesis of COVID-19 from a cell biology perspective. Eur Respir J 55(4):2000607. https://doi.org/10.1183/ 13993003.00607-2020

32. Hewitt J, Carter B, Vilches-Moraga A et al (2020) The effect of frailty on survival in patients with COVID-19 (COPE): a multicentre, European, observational cohort study [published online ahead of print, 2020 Jun 30]. Lancet Public Health S24682667(20)30146-8. https://doi.org/10.1016/S2468-2667(20)301468

33. Esposito S, Noviello S, Pagliano P (2020) Update on treatment of COVID-19: ongoing studies between promising and disappointing results. Infez Med 28(2):198-211

34. Zhong J, Tang J, Ye C, Dong L (2020) The immunology of COVID-19: is immune modulation an option for treatment?. The Lancet Rheumatology 2:e428-e436. https://doi.org/10.1016/ S2665-9913(20)30120-X

35. Şimşek Yavuz S, Ünal S (2020) Antiviral treatment of COVID-19. Turk J Med Sci 50(SI-1):611-619

Publisher's note Springer Nature remains neutral with regard to jurisdictional claims in published maps and institutional affiliations. 